\title{
Production of biomass and recombinant human-like collagen in Escherichia coli processes with different $\mathrm{CO}_{2}$ pulses
}

\author{
Wen J. Xue · Dai D. Fan · Longan Shang • \\ Chen H. Zhu $\cdot$ Xiao X. Ma $\cdot$ Yuan Y. Yu
}

Published online: 23 January 2009

(C) Springer Science+Business Media B.V. 2009

Erratum to: Biotechnol Lett (2009) 31:221-226

DOI 10.1007/s10529-008-9852-9

Panels $\mathrm{b}$ and $\mathrm{c}$ in Fig. 2 of the original publication were incorrect. The correct Fig. 2 is shown on the following page of this erratum.

The online version of the original article can be found under doi:10.1007/s10529-008-9852-9.

W. J. Xue · D. D. Fan $(\bowtie) \cdot$ C. H. Zhu

X. X. Ma . Y. Y. Yu

Department of Chemical Engineering and Shaanxi Key, Laboratory of Degradable Biomedical Materials,

Northwest University, Xi' an 710069, China

e-mail: fandaidi@nwu.edu.cn

D. D. Fan · L. Shang · C. H. Zhu · X. X. Ma

Shaanxi R\&D Center of Biomaterials and Fermentation

Engineering, Xi' an 710069, China

L. Shang

College of Biological and Chemical Engineering,

Ningbo Institute of Technology, Zhejiang University,

Ningbo 315100, China 

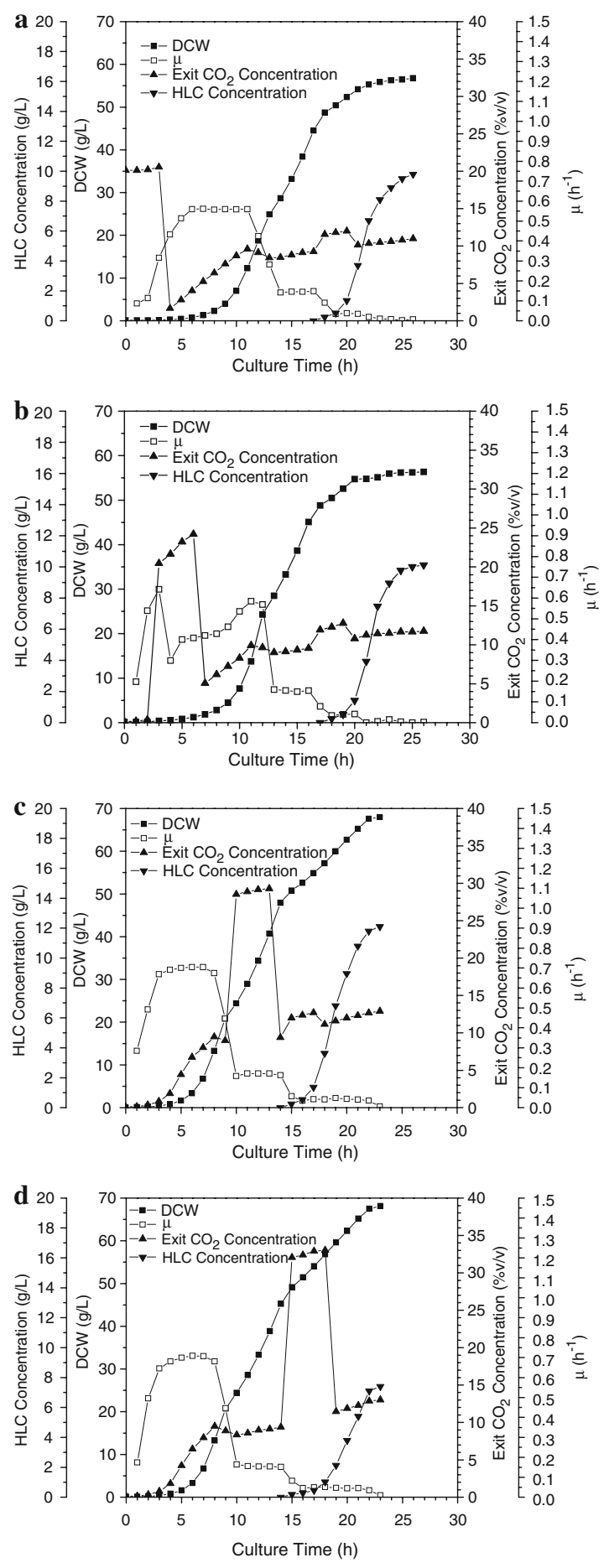

Fig. 2 Recombinant E. coli fermentation for HLC production under a $3-\mathrm{h} \mathrm{CO}_{2}$ pulse of $20 \%$ concentration injected at: a the lag phase; b the post-lag phase of batch culture; c the preinduction phase of fed-batch culture; d the post-induction phase of fed-batch culture. $\mathbf{\square}$, DCW; $\square$, Cell specific growth rate; $\boldsymbol{\Delta}$, Exit $\mathrm{CO}_{2}$ concentration; $\boldsymbol{\nabla}$, HLC concentration 